\title{
Probabilistic Estimation of Respiratory Rate from Wearable Sensors
}

\author{
Marco A.F. Pimentel ${ }^{1}$, Peter H. Charlton ${ }^{1,2}$, and David A. Clifton ${ }^{1}$ \\ 1 Institute of Biomedical Engineering, Department of Engineering Science, \\ Old Road Campus Research Building, University of Oxford, Roosevelt Drive, \\ Oxford, OX3 7DQ, UK \\ \{marco.pimentel, david.clifton\}@eng.ox.ac.uk \\ 2 Department of Biomedical Engineering, King's College London, London, SE1 \\ $7 \mathrm{EH}, \mathrm{UK}$ \\ peter.charlton@gstt.nhs.uk
}

\begin{abstract}
Respiration rate $(\mathrm{RR})$ is a physiological parameter that is typically used in clinical settings for monitoring patient condition. Consequently, it is measured in a wide range of clinical scenarios, notably absent from which is measurement using wearable sensors. With increasing numbers of patients being monitored via wearable sensors, as described below, there is an urgent need to be able to estimate RR from such sensors in a robust manner. In this chapter, we describe a novel technique for measuring $R R$ using waveform data acquired from wearable sensors.

The technique derives RR from a physiological signal which is routinely acquired by many mobile sensors: the photoplethysmogram (PPG). Each $\mathrm{RR}$ measurement from the proposed method is accompanied by a confidence measure, providing estimates of clinical quality that will allow the system to, for example, only report $\mathrm{RR}$ values when they exceed some probabilistic level of certainty. The goal of this method is to improve upon existing methods, which simply report $\mathrm{RR}$ values without probabilistic estimation, and which therefore suffer the lack of robustness that prevents their use in clinical practice.
\end{abstract}

\section{Introduction}

The value of measuring $\mathrm{RR}$ is demonstrated by its use in a wide range of clinical scenarios. It is routinely measured from acutely-ill patients in the emergency department [6], the intensive care unit [14, and in hospital ward settings [29]. Furthermore, RR is used to assist in the diagnosis of specific diseases, including pneumonia [13, and sepsis, 34, and with physiological conditions such as hypercarbia [5] and pulmonary embolism [9]. Measurement of RR using mobile sensors in hospitals would allow this clinically-significant quantity to be measured more frequently, allowing earlier identification of changes which may be indicative of acute deterioration. In the monitoring of patients in their own home, robust estimation of RR would assist with the 

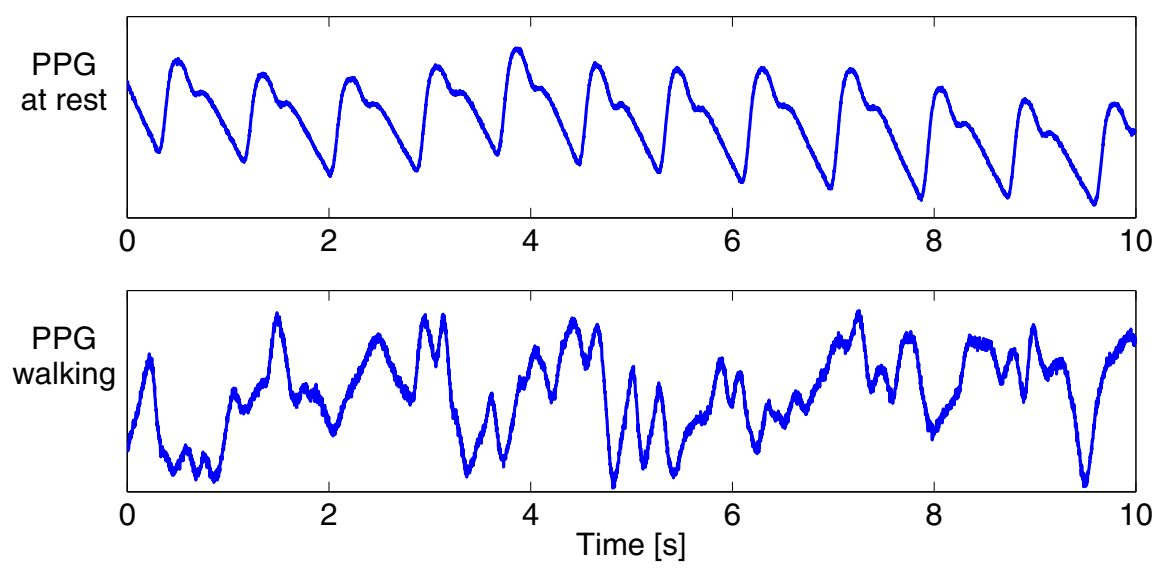

Fig. 1. PPG signals recorded from a healthy volunteer at rest and whilst walking, demonstrating the susceptibility of signals acquired using mobile sensors to movement artefact

management and monitoring of chronic diseases and postoperative rehabilitation [27. Despite the importance of RR in assessing the physiological state of ambulatory patients, mobile sensors do not commonly measure RR.

A potential solution is to estimate $R R$ from a physiological signal that is both routinely acquired by mobile sensors, and which is modulated by respiration. The electrocardiogram (ECG), PPG, and torso-mounted accelerometry signals are three such signals. All three signals are already acquired by mobile sensors for other purposes: (i) the ECG is used to monitor the heart rate, and to detect cardiac arrhythmias; (ii) the $\mathrm{PPG}$ is acquired during pulse-oximetry monitoring for measuring arterial blood oxygen saturation; and (iii) the accelerometry signal may be used for activity classification. In this paper, we consider the PPG signal for demonstrating the utility of our proposed probabilistic technique for estimating RR.

The strength of this probabilistic approach is that it provides RR estimates accompanied by a confidence measure. This is of particular utility for two reasons: firstly, a measure of the confidence of an RR estimate can be used to mitigate against the effect of noise on the final RR estimate. This is particularly relevant in the ambulatory setting where signals are highly susceptible to noise due to movement artefact, as illustrated in Figure 1, or to sensors becoming partially detached. Secondly, individual estimates from multiple sources or sensors can be subsequently fused according to their associated confidence measures, leading to a more robust final estimate. The latter is important for ensuring that the technique can be used widely, with varying patient groups, since the level of respiratory modulation of physiological signals may differ between those groups. 


\section{Extraction of Respiratory Rate from Wearable Sensors}

A plethora of techniques have been developed to estimate RR from PPG, ECG and accelerometry signals. In this section, we firstly describe the physiological mechanisms by which these signals are modulated by respiration. Secondly, the structure of algorithms for estimation of RR from these signals is presented. Finally, techniques which have been commonly used in algorithms for the estimation of RR are described.

\subsection{Physiological Basis of Respiratory Modulation}

Accelerometers can be used to capture signals modulated by respiration when appropriately positioned over the diaphragm [10. The accelerometer's orientation is changed by chest wall expansion and contraction during respiration. Thus, the direction of the force due to gravity acting on the accelerometer changes with respiration, modulating the signal.

In contrast, several different physiological mechanisms cause modulation of the PPG and ECG with respiration. We have previously described the mechanisms by which respiration modulates the PPG [21], although these are not fully understood. It is influenced by changes in venous return and stroke volume caused by changing intrathoracic pressure during respiration, changes in tissue volume due to varying arterial pressure, and respiratory sinus arrhythmid 1 . These result in amplitude modulation $(\mathrm{AM})$, baseline wander (BW, also known as respiratory-induced intensity variation), and modulation of beat-to-beat intervals (frequency modulation, FM), respectively. The ECG is modulated by respiration 3 due to: (i) changing orientation of the electrical axis of the heart relative to the electrodes; (ii) changing thoracic impedance (although this is secondary to changes in axis orientation); and, (iii) RSA. The first two mechanisms result in AM and BW, while the latter results in FM. The respiratory modulations exhibited by ECG and PPG signals are illustrated in Figure 2

The magnitude of each respiratory modulation may differ between patients. For instance, [17] evaluated the correlation of each modulation of the PPG with the respiratory cycle, and showed that no modulation is consistently optimal for respiratory monitoring across subjects. The correlation performance was found to be dependent on many factors including gender, age and body position. This finding suggests that, where multiple modulations are present in the PPG and ECG, more robust estimation of RR could be achieved by harnessing the information available provided by each modulation.

\footnotetext{
${ }^{1}$ RSA, which is variation in heart rate with respiration 15
} 

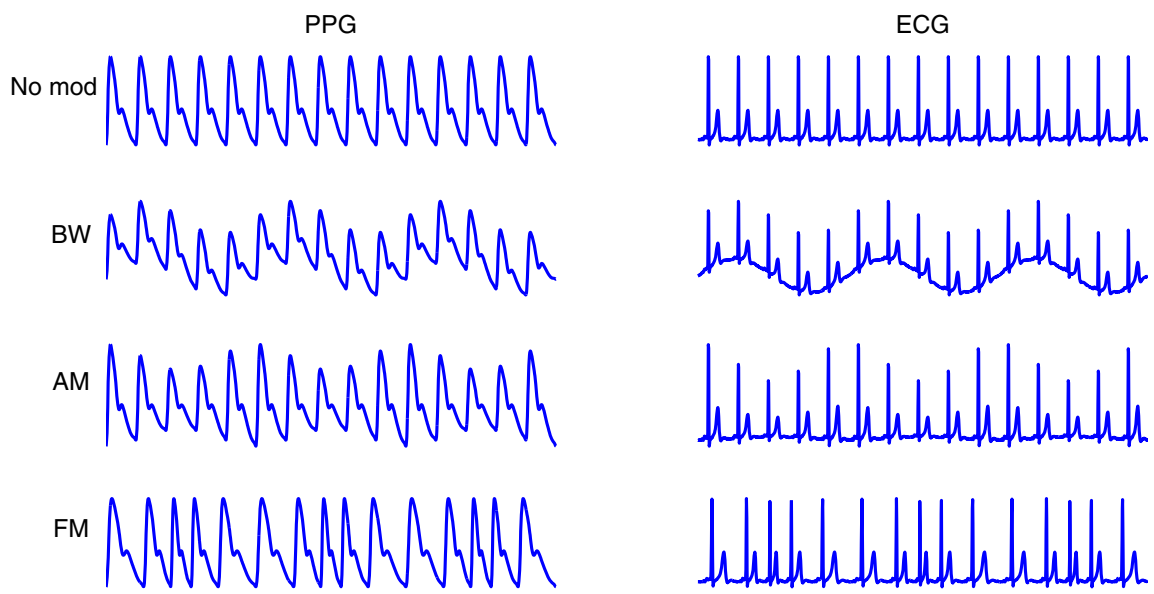

Fig. 2. Idealised respiratory modulations of the PPG (left hand side) and ECG (right hand side). During three respiratory cycles, from top: no modulation, baseline wander (BW), amplitude modulation (AM), and frequency modulation (FM). Adapted from [1.
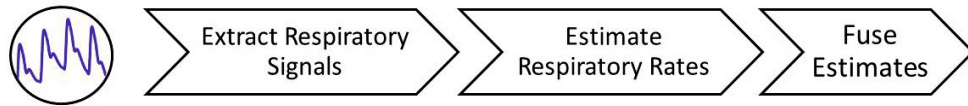

Fig. 3. The key components of an algorithm for estimating RR

\subsection{The Structure of Algorithms for Estimating Respiratory Rate}

Many algorithms for estimating RR from mobile sensor signals have been proposed in the literature. The algorithms consist of three key components, as illustrated in Figure 3. The role of each component is as follows:

- Extract Respiratory Signals: Time series exhibiting respiratory modulation are extracted from the raw physiological signal;

- Estimate RRs: Estimate a RR from each respiratory signal;

- Fuse RR Estimates: If multiple RR estimates are obtained, then these can be fused to obtain one estimate.

\subsection{Extraction of Respiratory Signals}

The first component of an algorithm for estimation of $R R$ is extraction of a respiratory signal, or signals. This extracts the respiratory modulation from 
the more complex, raw signal. For instance, a tri-axial accelerometer provides three measurements of the acceleration of a device using an orthogonal axis system. The component required for analysis is the direction of the acceleration due to gravity in the accelerometer's co-ordinate system, which is modulated by respiration. To obtain a respiratory signal, firstly a single, timevarying, acceleration vector is derived from the three measurements. Secondly, the rotation angle of the acceleration vector about the time-averaged predominant axis of rotation is calculated [4. Lastly, the respiratory signal is given by the angular velocity - the rate of change of the rotation angle with respect to time. The predominant axis of rotation is calculated over an extended time period to reduce the effect of noise, which is of particular importance in mobile monitoring.

Several techniques have been proposed for extracting respiratory signals from the ECG and PPG. The techniques can often be applied to both signals since they are both primarily cardiac in origin, with secondary respiratory modulations of much lower magnitude. The techniques fall into two categories: feature- and filter-based extraction techniques. Feature-based extraction of a respiratory signal consists of the extraction of a time series of beat-by-beat feature measurements. Beat detection is typically performed using a segmentation algorithm (such as that proposed by [16] for PPG signals or that proposed by [26 for ECG signals). AM is commonly extracted by measuring the pulse peak-to-trough amplitude; FM is extracted by measuring beat-to-beat intervals (between consecutive fiducial points such as pulse peaks), and a composite of AM and BW is extracted by measuring pulse peak amplitudes. Filter-based extraction consists of filtering the raw signal to attenuate non-respiratory frequency components. BW is commonly extracted using band-pass filtering to eliminate frequencies outside the range of plausible respiratory frequencies. A respiratory signal influenced by all three modulations has been extracted by filtering using the centred correntropy function $(\mathrm{CCF})$. This function incorporates information from both the time structure and statistical distribution of a signal. Exemplary signals extracted using feature- and filter-based approaches are shown in Figure 4.

\subsection{Estimation of Respiratory Rate}

Several methods have been proposed for estimating the RR from a window of data from a respiratory signal. This can be performed in both the timeand the frequency-domain, although frequency-domain techniques are most commonly used, four of which are now described.

Frequency-domain techniques typically require evenly sampled data. Consequently, respiratory signals derived using feature-based analysis must usually be re-sampled onto an even grid prior to frequency-domain analysis (see Figure 6). 


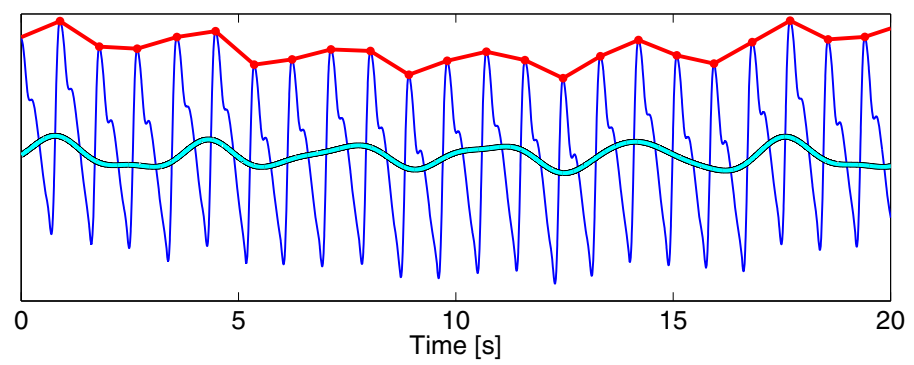

Fig. 4. Comparison of feature- and filter-based techniques for extraction of respiratory signals. Baseline wander (BW) signals have been extracted from the PPG using a feature-based technique (red) in which pulse peak amplitudes are extracted, and a filter-based technique (cyan) in which a band-pass filter with cut-off frequencies of 0.2 and $0.6 \mathrm{~Hz}$ (12 and $36 \mathrm{rpm})$.

Fourier analysis has been used to calculate the frequency spectra of respiratory signals, from which the RR can be estimated 8 . However, this technique does not perform well when RR is non-stationary across the window of data analysed. The short-time Fourier transform (STFT) has been proposed to improve the accuracy of RR estimation in the presence of non-stationarity 32. In their application of the technique, Shelley et al. analysed the raw PPG signal using the STFT and a moving Hann window of $82 \mathrm{~s}$ duration. This approach is illustrated in Figure 5.

Autoregressive (AR) modelling has been used to identify the resonant frequencies contained within a respiratory signal. The poles generated by the ("all-pole" filter) model correspond to resonant frequencies, where the frequency is determined by the pole's phase angle. The respiratory pole (and accompanying frequency) can be identified as the pole with the greatest magnitude within the plausible range of respiratory frequencies. Alternatively, AR modelling can be used to calculate the power spectral density (PSD) of a respiratory signal. This is related to the analysis of the model's poles, since each pole provides a contribution to the PSD, centred on its resonant frequency 20]. The size of this contribution is determined by the pole's magnitude.

For all techniques except analysis of the poles of an AR model, a frequency spectrum is obtained. The respiratory frequency is typically identified from the spectrum as the frequency corresponding to the maximum spectral power.

\subsection{Fusion of Respiratory Rate Estimates}

The final step of estimating the RR from a physiological signal is to fuse estimates derived from multiple respiratory signals. Two recently proposed methods are considered here. The simplest method of fusing multiple RR estimates is to calculate the average. Karlen et al. used this approach, 


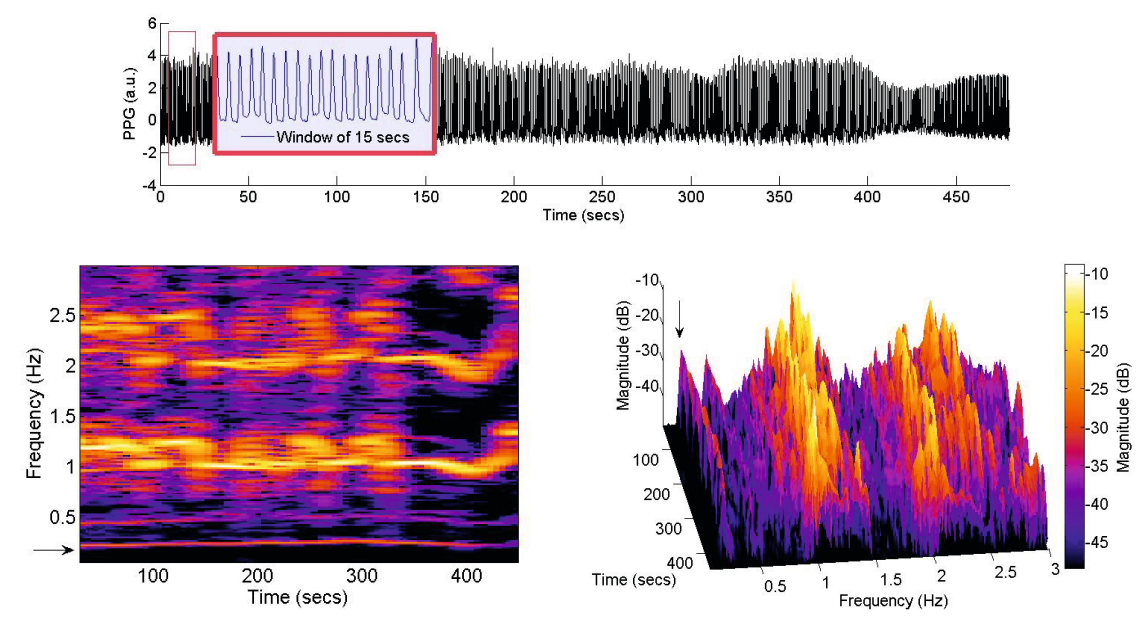

Fig. 5. Example of a full 8-minute record of PPG (on top) used for RR estimation (a 15-second segment of the signal is highlighted as indicated in the red box). The time-frequency spectrum, calculated using the short-time Fourier transform (STFT), is represented on the bottom-left. The frequency band corresponding to the respiratory rate (indicated by an arrow) is observed (at $0.18 \mathrm{~Hz}=10.8 \mathrm{rpm}$ ), and is practically constant throughout the record. The corresponding magnitude of this band may be better observed in the 3D representation of the time-frequency spectrum on the bottom-right. This also shows the overwhelming signal strength for the heart rhythm at approximately $1.1 \mathrm{~Hz}$.

obtaining the final estimate as the mean of the individual estimates [11. They also extended this approach by including a quality assessment check. Any set of estimates with a standard deviation of more than $4 \mathrm{rpm}$ was eliminated, and no RR estimate was obtained from that window of data.

A further method was proposed by Orphanidou et al. , which fuses RR estimates derived from the poles of an AR model 25. Poles outside of 0.1 - $0.6 \mathrm{~Hz}(6-36 \mathrm{rpm})$ are excluded from the analysis. The remaining pole with the greatest magnitude is identified for each respiratory signal, and any poles which have a magnitude of less than $95 \%$ of this pole's magnitude are rejected. The candidate respiratory pole for each signal is then identified as the pole with the lowest corresponding frequency. The final RR estimate is the frequency corresponding to the candidate pole with the greatest magnitude.

\section{A Probabilistic Approach}

The main drawback of the approaches discussed above is that they provide a point estimate of the respiratory rate. The uncertainty associated with the 

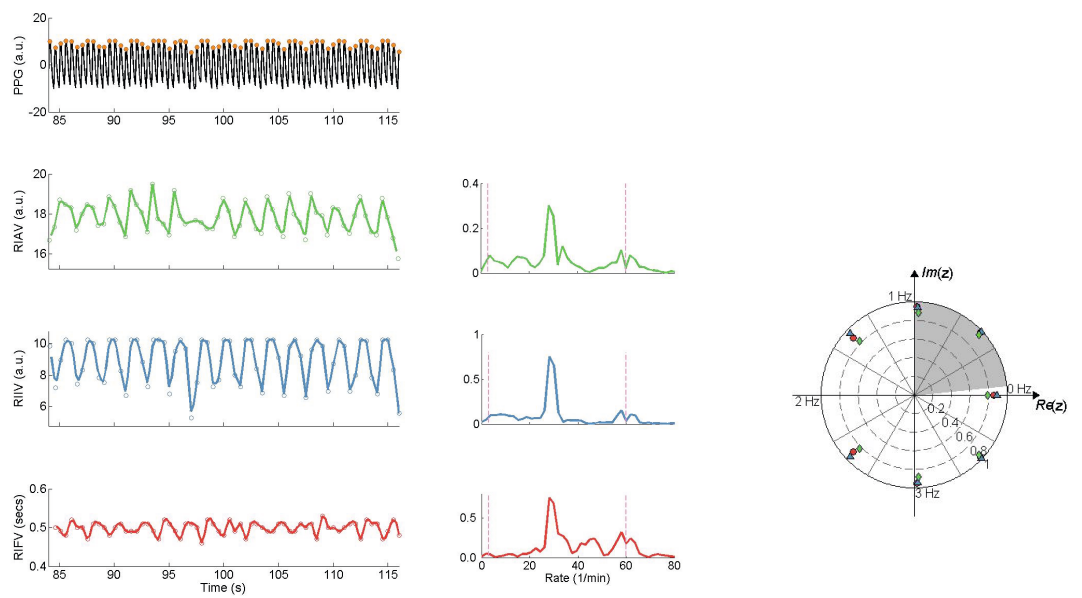

Fig. 6. Example of a 32 secs PPG sliding window (represented with black line on the top-left corner) used for RR estimation using two different methods. RIAV (top, in green), RIIV (middle, in blue), and RIFV (bottom, in red) are extracted and resampled to $4 \mathrm{~Hz}$ (left column). In the method proposed by [11, the power spectrum is calculated for each modulation using FFT (middle column), and the maximum power is selected within a physiologically expected $R R$ range (dashed vertical lines). In the method proposed by 25, the poles from each modulation are determined using an autoregressive model, order $p=7$ (right column), and the dominant pole within the possible range of RR (shown in grey) gives the "fused" estimate.

estimated value cannot be directly quantified, due to the nature of the algorithms employed. Specifically, the "fusion" approach of the estimates derived from the different modulations lack validity, and may reduce significantly the number of windows for which a final estimate of RR is computed [11, 8].

The failure of existing methods to estimate RR accurately using data from actual patients, rather than from healthy volunteers, motivates the use of a probabilistic approach. We propose a method that uses the framework of Gaussian process regression to extract RR from the different sources of modulation in the PPG signal. This brings all of the advantages of a principled, probabilistic approach: our uncertainty in the estimation may be directly quantified; incompleteness, noise, and artefact may be handled in a robust manner; and the output may consist of a predictive posterior distribution, rather than a single estimate - this is useful if the estimate of the respiratory rate is to be used as the input to a subsequent probabilistic inference system, where knowing the full distribution of the input is more informative than a point estimate. Finally, due to the generative nature of the approach, it is possible to generate data from the model, which can be useful for both 
estimating the behaviour of RR during periods of missing data, and making predictions.

\subsection{Gaussian Process Regression}

In this section, we provide a brief summary of Gaussian processes for regression. It therefore makes a rather compressed introduction to the topic. A more thorough introduction is available in 28 .

When performing a regression task we assume there exists some optimal prediction function $f \in \mathcal{X} \rightarrow \mathcal{Y}$, possibly with a noise distribution. In linear regression, we may assume that the outputs $y$ are a linear function of the inputs $\mathbf{x}$, with some parameters $\theta$, often much smaller than the number of training examples $N$, such that $|\theta| \ll N$. However, for many real-world data sets a simple parametric form, such as a linear form, is an unrealistic assumption. Therefore, we would like to have models that can learn general functions $f$. Since the functions may not be summarised by a small (fixed) number of parameters $\theta$, maximum likelihood estimation of the parameters typically causes severe overfitting. Therefore, in order to perform inference and make predictions in a probabilistic framework, we must place a prior probability distribution on functions. We make predictions using our posterior on an underlying predictive function $f$ given a set of training examples in the form of input-output pairs: $\mathcal{D}=\left\{\left(\mathbf{x}_{i} \in \mathbb{R}^{D}, y_{i} \in \mathbb{R}\right)\right\}_{i=1}^{N}$.

Gaussian processes provide a distribution over real-valued functions which is widely used for non-linear regression and classification tasks [28. By definition, a function $f: \mathcal{X} \rightarrow \mathbb{R}$ is distributed according to a Gaussian process if and only if $p\left(f\left(\mathbf{x}_{1}\right), \ldots, f\left(\mathbf{x}_{N}\right)\right)$, the density of those function's values at any $N$ points $\mathbf{x}_{i} \in \mathcal{X}$, is jointly Gaussian. This allows Gaussian processes to be tractably parameterised by a mean function $m(\mathbf{x})$ and a covariance kernel function giving the entries of matrix $\mathbf{K}\left(\mathbf{x}_{i}, \mathbf{x}_{j}\right)$ and which specifies the correlations within any finite point set. This yields

$$
\mathbf{y}=f(\mathbf{x}) \sim \mathcal{G P}\left(m(\mathbf{x}), \mathbf{K}\left(\mathbf{x}_{i}, \mathbf{x}_{j}\right)\right)
$$

with possibly some Gaussian observation noise. Note that the covariance matrix $\mathbf{K}$, or Gram matrix, whose entries $\mathbf{K}_{i j}$ are often thought of as the "similarity" between inputs $\mathbf{x}_{i}$ and $\mathbf{x}_{j}$, encodes our prior knowledge concerning the functional behaviour we wish to model.

Without loss of generality, the prior mean function is typically set to zero: $m(\mathbf{x})=0$. A commonly-used covariance function is the squared-exponential,

$$
k_{S E}\left(\mathbf{x}_{i}, \mathbf{x}_{j}\right)=\sigma_{0}^{2} \exp \left(-\frac{\left\|\mathbf{x}_{i}-\mathbf{x}_{j}\right\|^{2}}{2 \ell^{2}}\right),
$$

where $\sigma_{0}$ and $\ell$ are hyperparameters modelling the $y$-scaling and $x$-scaling (or time-scale if the data are timeseries), respectively, and where $\|\cdot\|$ 
(a)

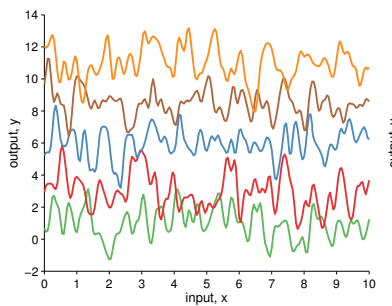

(d)

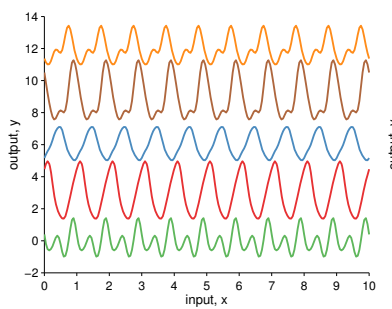

(b)

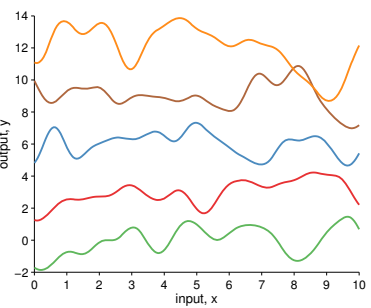

(e)

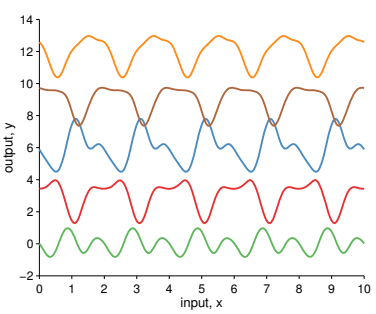

(c)

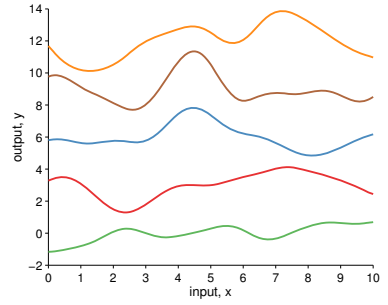

(f)

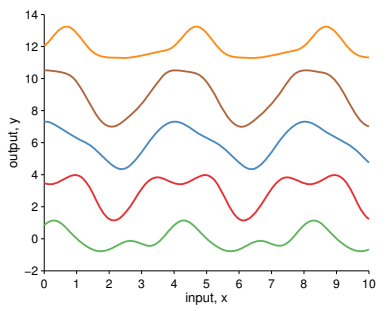

Fig. 7. (a-c) Functions drawn from a Gaussian process with a squared-exponential covariance function (zero-mean) with $\sigma_{0}=1$ and $\ell=0.1$ (a), 0.5 (b), and 1 (c); (d-f) Functions drawn from a Gaussian process with a periodic covariance function (zero-mean) with $\sigma_{0}=1, \ell=0.5$, and $T=1(\mathrm{~d}), 2(\mathrm{e})$, and 4 (f).

denotes the Euclidean norm. The squared-exponential covariance function is said to be stationary because it only depends on the difference between points $\mathbf{x}_{i}-\mathbf{x}_{j}$ (see Figure 7). In general, covariance functions have to fulfill Mercer's theorem, meaning that $\mathbf{K}\left(\mathbf{x}_{i}, \mathbf{x}_{j}\right)$ has to be symmetric and positive semidefinite, and therefore $k_{S E}(\cdot, \cdot)$ is a valid kernel. Many mathematical operations, such as the summation or the product, preserve positive definiteness and can therefore be used for combining "old kernels" to make "new kernels". Also, note that a valid covariance function under any arbitrary (smooth) map remains a valid covariance function. An interesting example of this mapping is given in [19, where the one-dimensional input variable $x$ is mapped to the two-dimensional $\mathbf{u}(x)=(\cos (x), \sin (x))$ to give rise to a periodic random function of $x$. Using the squared-exponential kernel in $\mathbf{u}$-space, yields

$$
k_{P e r}\left(x_{i}, x_{j}\right)=\sigma_{0}^{2} \exp \left(-\frac{1}{2 \ell^{2}} \sin ^{2}\left(\pi\left|\frac{x_{i}-x_{j}}{T}\right|\right)\right),
$$

Here, the "extra" hyperparameter $T$ corresponds to the period (see Figure 7). A survey of several covariance functions is given in [28, Chapter 4]. 
Given a training set $\mathcal{D}$, using the standard conditioning rules for a Gaussian distribution, we can obtain the predictive distribution on a new observation $y_{*}$ at test input $\mathbf{x}_{*}$ :

$$
\left[\begin{array}{c}
\mathbf{y} \\
y_{*}
\end{array}\right]=\mathcal{N}\left(\left[\begin{array}{l}
0 \\
0
\end{array}\right],\left[\begin{array}{cc}
\mathbf{K} & \mathbf{K}_{*} \\
\mathbf{K}_{*}^{\top} & \mathbf{K}_{* *}
\end{array}\right]\right)
$$

implying

$$
\begin{aligned}
p\left(y_{*} \mid \mathbf{x}_{*}, \mathbf{X}, \mathbf{y}\right) & \sim \mathcal{N}\left(\mu_{*}, \sigma_{*}^{2}\right), \text { with } \\
\mu_{*} & =\mathbf{K}_{*}^{\top} \mathbf{K}^{-1} \mathbf{y} \in \mathbb{R}, \\
\sigma_{*}^{2} & =\mathbf{K}_{* *}-\mathbf{K}_{*}^{\top} \mathbf{K}^{-1} \mathbf{K}_{*} \in \mathbb{R}^{+} .
\end{aligned}
$$

Here, $\mathbf{K}_{*}=k\left(\mathbf{X}, \mathbf{x}_{*}\right) \in \mathbb{R}^{N+1}$ is the cross-covariance between the test input $\mathbf{x}_{*}$ and the training inputs $\mathbf{X} ; \mathbf{K}_{* *}=k\left(\mathbf{x}_{*}, \mathbf{x}_{*}\right) \in \mathbb{R}^{+}$is the prior variance of the test point.

The values of the hyperparameters $\boldsymbol{\theta}$ may be optimised by, for example, minimising the negative log marginal likelihood (NLML) which is defined as

$$
\begin{aligned}
\mathrm{NLML} & =-\log p(\mathbf{y} \mid \mathbf{x}, \boldsymbol{\theta}) \\
& =\frac{1}{2} \log |\mathbf{K}|+\frac{1}{2} \mathbf{y}^{\top} \mathbf{K}^{-1} \mathbf{y}+\frac{N}{2} \log (2 \pi)
\end{aligned}
$$

This is sometimes called the type-II maximum likelihood (if we remove the negative logarithm). Interpreting the NLML as a cost function reveals that the first term penalises model complexity and the second term penalises low data likelihood (i.e., low data fitness). Bias-variance trade-off is therefore performed by minimising the NLML, which is commonly achieved using gradient descent.

A full Bayesian treatment of GP regression requires integration over the posterior distribution of the hyperparameters. Even though most calculations in the GP regression framework are analytically tractable, the integral over the posterior of the hyperparameters often is not 28. The integration over the posterior of the hyperparameters $p(\boldsymbol{\theta} \mid \mathcal{D})$, with $\boldsymbol{\theta}=\left\{\sigma_{0}, \lambda, P_{L}, \varepsilon\right\}$, can be approximated by a point via the maximum a posteriori (MAP) estimate

$$
\begin{aligned}
\hat{\boldsymbol{\theta}} & =\arg \max _{\boldsymbol{\theta}} p(\boldsymbol{\theta} \mid \mathcal{D}) \\
& =\arg \min _{\boldsymbol{\theta}}[-\log p(\mathcal{D} \mid \boldsymbol{\theta})-\log p(\boldsymbol{\theta})]
\end{aligned}
$$

In this approximation, the distribution over the hyperparameters is assigned a point mass at the mode of the posterior, allowing the marginal distribution of the latent function to be approximated by $p(f \mid \mathcal{D}) \approx p(f \mid \mathcal{D}, \hat{\boldsymbol{\theta}})$. This approach is computationally attractive. The grid search approximation 
to the full integral over the posterior distributions of the hyperparameters follows the work of Rue et al. [30, in which the posterior mode $\hat{\theta}$ is first located by maximising the log-posterior distribution $\log p(\theta \mid y)$, and the shape of the log-posterior is approximated with a Gaussian, the covariance of which is the inverse of the negative Hessian at the mode (more details may be found in [28, 30]).

\subsection{Proposed Method}

Using the Gaussian process framework described above, we propose a method for "fusing" the estimates from the different modulations exhibited by the PPG. In the first step, for a given window of the signal, the (unevenlysampled) time-series data corresponding to the BW, AM, and FM are extracted using a segmentation algorithm (note that no interpolation is performed). A Gaussian process with a periodic covariance function is then fitted to each of the timeseries, using the procedure described above to obtain an estimate of both the value and uncertainty of the respiratory rate value (which is determined directly from the distribution over the period, $T$ ). After converting the period $T$ to $\mathrm{RR}(60 / T)$, this results in three (uncertain) estimates of $\mathrm{RR}$, given by $\mu_{k} \pm \sigma_{k}$, with $k=\{1,2,3\}$.

In order to combine the three estimates into a final RR estimate $\mu$, a weighted average is employed (as described in [33]), as such

$$
\mu=\mu_{o} \pm\left(\sum_{k=1}^{3} w_{k}\right)^{-1 / 2}
$$

with

$$
\mu_{o}=\sum_{k=1}^{3} w_{k} \mu_{k} / \sum_{k=1}^{3} w_{k}, \quad \text { where } w_{k}=\frac{1}{\sigma_{k}^{2}} .
$$

Rather than the best estimate being given by the arithmetic mean of the RR estimates, the weighted average of the RR estimates is determined and taken as the final estimate of $\mathrm{RR}$ for that window. Less reliable estimates will have larger variances $\sigma_{k}^{2}$ and correspondingly smaller weights $w_{k}$.

\section{Materials and Methods}

In order to evaluate the performance of the proposed approach, described above, we have implemented state-of-the-art methods using the methods described according to each paper. The pre-processing stages are detailed be- 
low. The source code of the implementations of the methods described in this study (as well as the data sets used) are available for download? 2 .

\subsection{Datasets}

For the analysis described in this experiment we used two independent, publicly available datasets: the Capnobase benchmark datase+3, and a dataset extracted from the MIMIC II database 4 .

\subsubsection{CapnoBase Dataset}

The CapnoBase dataset, described in [12], consists of ECG and PPG recordings, and capnometry data, all recorded using a sampling frequency of 300 $\mathrm{Hz}$, from 59 children (median age: 8.7 , range: $0.8-16.5$ years) and 35 adults (median age: 52.4, range: 26.2-75.6 years). The cases in the dataset were randomly selected from a larger collection of physiological signals collected during elective surgery and routine anaesthesia. In the work reported in [11, the CapnoBase dataset was divided into a test set consisting of forty-two 8-minute segments (336 minutes in total) containing reliable recordings of spontaneous or controlled breathing, and a calibration set consisting of one hundred twenty-four 2-minute segments (248 minutes) from the remaining 52 cases. As in [11, the test dataset (forty-two 8-minute segments) was considered for analysis.

\subsubsection{MIMIC II Dataset}

The dataset extracted from the MIMIC II database 31] comprises PPG recordings and respiration signals acquired using the conventional impedance plethysmogram (IP), both sampled at $125 \mathrm{~Hz}$, from 53 adults (median age: 64.8, range: [19-90+], 32 females). The cases in the dataset were extracted from a larger cohort of patients who were admitted to medical and surgical intensive care units at the Beth Israel Deaconess Medical Center, Boston, MA. As in the previous dataset, 8-minute segments containing reliable recordings of spontaneuous breathing were randomly selected.

\subsection{Data Preparation}

The recordings from both databases were grouped into different age groups, according to the age of the patient from which the recordings were taken (see Figure 8). Forty-one 8-minute recordings from the CapnoBase database and fifty-two 8-minute recordings from the MIMIC II database were included in

\footnotetext{
${ }^{2}$ To appear at http://www.robots.ox.ac.uk/ davidc/

3 Available at http://www. capnobase.org

${ }^{4}$ Available at https://mimic.physionet.org/
} 


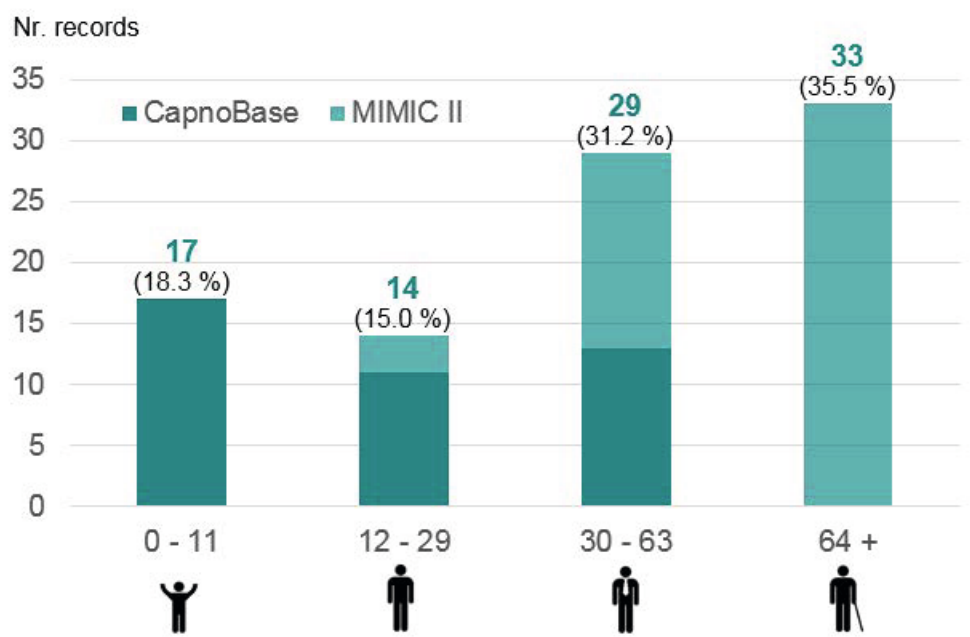

Fig. 8. Number of records available in the CapnoBase and MIMIC II databases for each age group

this analysis, as the age associated to two recordings (one from each database) was not available. All signals were re-sampled to $125 \mathrm{~Hz}$. For the work described in this study, respiratory rate was computed for a single window size: 32 -second windows, with successive windows having 29 seconds overlap. This window size was selected as it did not need zero padding (for frequencybased analysis) and was within reasonable physiological and clinical limits (as discussed in 17, 11]). For all methods described above, RR was estimated within the plausible range of respiratory frequencies set to $0.05-1 \mathrm{~Hz}$, or $3-$ $60 \mathrm{rpm}$. The estimated RR values from the PPG recordings were compared with the reference respiratory rate obtained from the reference gold standard recordings in each database.

In the CapnoBase dataset, the capnometric waveform was used as the reference gold standard recording for RR. Each breath in the capnogram has been manually labelled by a research assistant, and the annotations were used to derive the reference values. For each window, the time between consecutive exhalations and between consecutive inhalations was calculated. The median value was used to determine the reference $\mathrm{RR}$ for that window.

In the MIMIC II dataset, because of the absence of manually annotated data, we calculated the reference respiratory rate from the IP signal as follows. The respiration signal was first down-sampled to $4 \mathrm{~Hz}$ and then filtered using a 0.1-0.6 Hz finite-impulse response band-pass filter. For each window of the filtered signal, two RR estimates were calculated using two different algorithms. The first algorithm applied was a 3-point peak detector algorithm (outlined below), which was used to determine the first estimate of RR. 
The second algorithm involved converting the filtered signal to the frequency domain using the fast Fourier transform. The resulting power spectrum was analysed for the frequency with maximum power within the expected respiratory frequency range, and the second RR estimate was obtained. Only those window sections of data for which the agreement between both estimates (provided by the two algorithms) was within $2 \mathrm{rpm}$ were retained, and the mean value of the two estimates was taken as the reference RR. As a result, for a window size of 32 seconds, $93 \%$ of all available windows were deemed to be "valid". This approach ensured that only the highest quality reference values were considered by potentially eliminating regions of low IP signal quality.

\subsection{Methodology}

In our experiments, to extract the three respiratory-induced variations, PPG beat detection was performed using a segmentation algorithm proposed in [16], where a "beat" in this context corresponds to the pulse in the PPG associated with a heartbeat. This processing produces a series of maximum and minimum intensities for each pulse detected. The series of maximum intensities of the PPG pulses was used for extracting the BW timeseries. The amplitude of the resulting series of beats was determined (as the difference between the peaks and onsets of the beats) in order to derive the AM timeseries. The intervals between successive beats (which corresponds to the pulse period) was also calculated to extract the FM timeseries.

\subsubsection{Methods}

We compared the performance of the proposed approach with that of four other methods, which are described below.

Method 1. The first method is based on the approach proposed in [11, which, as described above, uses Fourier analysis to calculate the frequency spectra of the derived respiration signals. Because spectral analysis requires evenly sampled data, each timeseries (corresponding to BW, AM and FM) is first re-sampled onto an even $4-\mathrm{Hz}$ grid using linear interpolation. The frequency at which the maximum intensity of each spectrum is obtained, is taken as the respiratory frequency. The final RR estimate is obtained as the mean of the three estimates, and any set of estimates with a standard deviation of more than $4 \mathrm{rpm}$ is eliminated (i.e., no RR estimate is obtained from that window).

Method 2. This method is based on that described in [25]. After re-sampling the three timeseries onto an even $4-\mathrm{Hz}$ grid using linear interpolation, an AR model (of order 7) is fitted to each timeseries. The resonant frequencies corresponding to the poles generated by the models are then evaluated, and 
the respiratory is identified as that corresponding to the pole with the greatest magnitude within the plausible range of respiratory frequencies.

Method 3. In this method (based in [32]), the raw PPG signal is analysed using the STFT and a moving Hann window of 82 seconds, and the time-frequency spectrum is obtained. The frequency component (within the plausible range of respiratory frequencies) with the greatest magnitude in each window is identified as the RR.

Method 4. The fourth method implemented corresponds to the method used in [8, who proposed an algorithm based on the time-varying correntropy spectral density function applied (directly) to the PPG signal. From the correntropy spectral density applied to each signal segment, the heart rate is estimated by detecting the maximum frequency peak within the cardiac frequency band, and filtered from the signal (using a zero-phase $5^{\text {th }}$-order low-pass filter with a cutoff frequency of $0.1 \mathrm{~Hz}$ below the cardiac frequency). The $\mathrm{RR}$ is finally estimated by detecting the maximum frequency peak within the respiratory frequency band.

\subsubsection{Signal quality index}

In order to remove windows of poor PPG signal quality, we employed a signal quality algorithm. Signal quality of the original PPG waveform was assessed using the same method as proposed by Li et al. [18]. The overall signal quality is assessed using four measures: three based upon template matching and a fourth based upon flat-line detection. For the template methods, the same beat detector [16] was applied to the PPG signal. The first 10 beats in each signal are assumed to be good quality, and a template is created by averaging windows centered on these 10 beats. For each subsequent beat, the signal quality is assessed using the correlation between the current beat and the template. This correlation is calculated for 1) the original unmodified beat, 2 ) the beat after a linear detrending, and 3) the beat after dynamic time warping (DTW) [18. A rule-based approach for assigning signal quality using the above three signal qualities (SQIs 1-3) and the fourth signal quality based on flatline detection (SQI 4), as proposed by Li et al. [18, is used to aggregate the four metrics into a single estimate. The rule is as follows:

$$
S Q I= \begin{cases}\text { Excellent(E) } & \text { if } \text { All } 4 S Q I \geq 0.9 \\ & \text { if Any } 3 S Q I \geq 0.9 \\ \text { Acceptable(A) } & \text { if } \text { All } 4 S Q I \geq 0.7 \\ & \text { if median }\left(S Q I_{1}, S Q I_{2}, S Q I_{3}\right) \geq 0.8 \\ & \text { and } S Q I_{1} \geq 0.5 \text { and } S Q I_{4} \geq 0.7 \\ \text { Unacceptable(U) } & \text { otherwise }\end{cases}
$$


Windows with an "unacceptable" quality were removed, and no RR estimates were obtained for those windows (for any of the methods implemented in this experiment).

\subsubsection{Methods Evaluation}

The performances of the methods were assessed by calculating the mean absolute error (MAE) in breaths per minute $(\mathrm{rpm}), \mathrm{MAE}=\frac{1}{n} \sum_{i=1}^{n} \mid \hat{y}_{i}-$ $y_{\text {ref, } i} \mid$, where $n$ is the number of valid windows over each patient in each age-group, $\hat{y}_{i}$ is the estimated respiratory rate $\left(\mu_{o}\right.$ in the case of our proposed method) and $y_{r e f, i}$ is the reference respiratory rate for window $i$.

\section{Results}

The results obtained with each method are shown in Figure 9. Results are displayed as the average of MAEs for the records included in each age-group. Also, the average number of windows considered in each method are displayed; i.e., the percentage of windows that were not rejected, and, hence, for which an estimate of RR was obtained.

\section{Discussion}

$\mathrm{RR}$ is a valuable physiological parameter which is used in a wide range of clinical scenarios. However, it is not yet commonly measured by wearable sensors. In this chapter we have presented a novel probabilistic technique for estimation of RR from physiological signals which are already acquired by wearable sensors. The technique is set in the framework of Gaussian process regression, whereby each RR estimate is accompanied by a measure of its associated uncertainty. The benefit of quantifying the uncertainty associated with RR estimates is that it allows multiple estimates obtained simultaneously to be fused in a principled manner. The performance of this technique was assessed using data acquired from patients spanning a wide range of ages. The results presented suggest that the technique may confer particular benefit in patients over 30 years old. Further work is required to determine whether use of this technique in mobile sensors would confer clinical benefit.

We have described a generalised structure which can be used to decompose algorithms for estimating RR into their constituent components, based that described in [11. This structure allows algorithms to be adapted to estimate RR from any signal which is modulated by respiration. The first component, extraction of respiratory signals, should be adapted to the input signal. For instance, feature-based extraction methods should be tailored to the input signal to ensure correct beat detection and identification of fiducial points each cardiac cycle. However, once respiratory signals have been extracted, the remainder of an algorithm can be applied unchanged, regardless of the input 


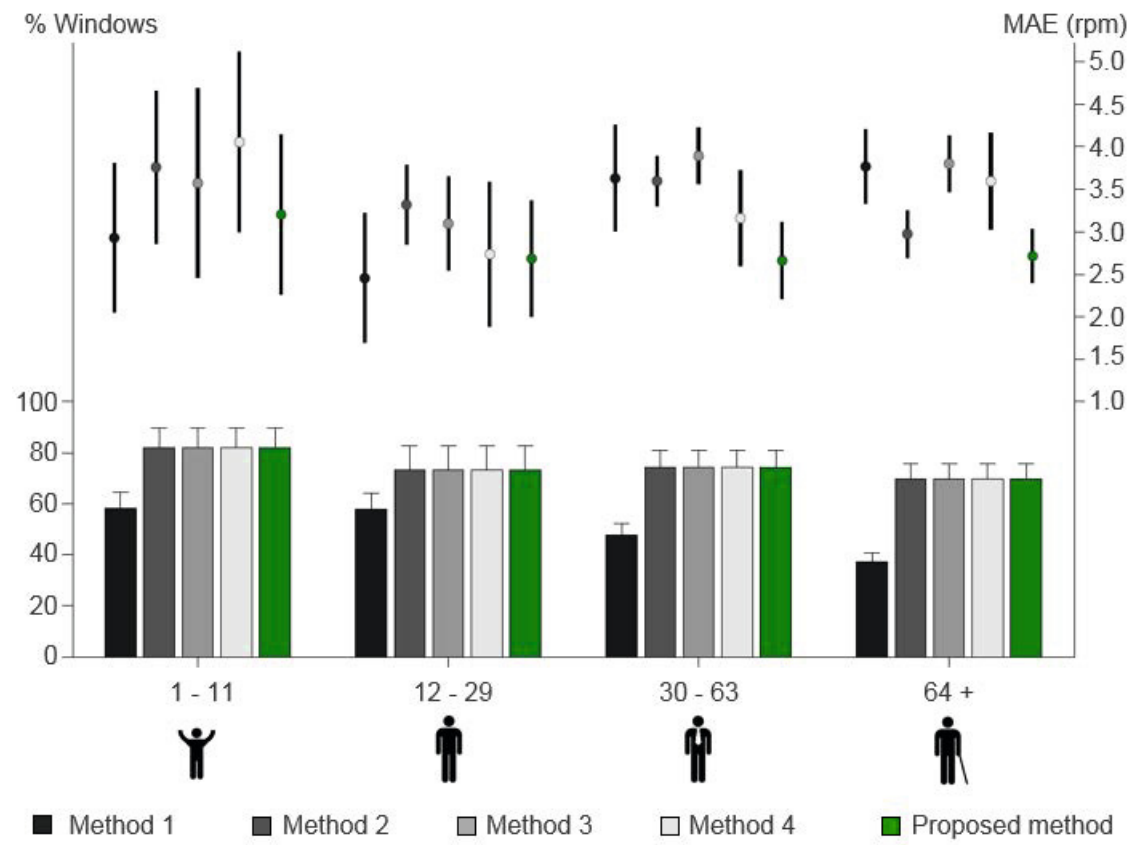

Fig. 9. Results obtained for the different methods: bars denote the average percentage of windows considered in each method (refer to axes on the left) and points denote the mean absolute error (refer to axes on the right). Error bars denote one standard error of the mean.

signal. The GP-based algorithm presented here uses a common approach for extraction of respiratory signals corresponding to BW, AM and FM, as described in [11. The remainder of the algorithm, in which RR estimates are obtained from each respiratory signal, and multiple estimates are fused to provide a single estimate, could be applied to other signals acquired by wearable sensors such as ECG and accelerometry signals.

The proposed method of fusing multiple RR estimates based on their uncertainties has been designed to facilitate robust estimation of RR across a wide range of patient groups. The three respiratory modulations of the ECG and PPG are caused by physiological mechanisms which are likely to be affected by the physiological state of patients. Consequently, the magnitude of each modulation is likely to differ between patient groups. For instance, heart rate variability is known to diminish with age 24]. It may therefore be postulated that RSA, the physiological mechanism which causes FM, being closely linked to heart rate variability may also diminish with age. Furthermore, chest expansion is known to be affected by both age and gender [22]. Similarly, this physiological change may be expected to affect the magnitudes 
of BW and AM. The effect of physiological factors on the magnitudes of respiratory modulations remains an open research question [23. However, it is clear that if the magnitudes of modulations do vary between patients, then an adaptable method for fusing estimates derived from each modulation is required. Indeed, the results suggest that this novel technique confers greatest benefit in those patients aged over 30, which may be due to its ability to fuse RR estimates according to their associated uncertainties.

We see three potential directions for future work to translate this novel technique into clinical practice. Firstly, the approach to signal quality assessment, performed here using a signal quality algorithm, could be enhanced. Simply put, signal quality algorithms are designed to determine whether an input signal has a high signal to noise ratio. However, the signal investigated by the algorithm used with this technique is the cardiac, rather than respiratory, signal. An additional step may be required to exclude any signal in which there is insufficient respiratory modulation to estimate RR.

The second potential improvement lies in the methodology used to extract respiratory signals. This technique uses the feature-based methods for extraction of BW, AM and FM proposed in [11. However, this results in respiratory signals of a low sampling frequency of one sample per cardiac cycle. Furthermore, they are calculated using one or two fiducial points in each cardiac cycle. Considering that signals are often acquired from mobile sensors at sampling frequencies of $75 \mathrm{~Hz}$ and above, much of the available information has not been included in the calculation of the respiratory signal. In addition, the cardiac frequency is approximately three to five times higher than the respiratory frequency. Therefore, relatively few samples of a single respiratory cycle are acquired. In contrast, the alternative category of methods, filter-based methods, uses all the available information and provides a respiratory signal at the sampling frequency of the original signal (as illustrated in Figure 4).

Thirdly, wearable sensors must be suitable for use by a wide range of patient groups. To this end the performance of wearable sensor technology must be tested across a heterogeneous patient cohort which is representative of the end users. In this study data collected from subjects with a wide range of ages was used, from paediatric to elderly patients. The only significant age cohort omitted was the neonatal cohort. This is in keeping with recent work by Addison et al. 2, who published a study of a RR algorithm for use with the PPG which was tested on data acquired from adult patients across the general care floor with a wide range of medical conditions and ages. This study is limited, however, by not including data from patients outside of the hospital setting, and by using data acquired from patients whilst being stationary, rather than truly ambulatory. 


\section{Conclusions}

We proposed and evaluated a novel fusion algorithm for the extraction of respiratory rate from waveforms acquired by non-invasive wearable sensors; in this case, the PPG signal. This work has demonstrated that it is possible to enhance the capabilities of wearable devices (such as the pulse oximeter) by providing the possibility of incorporating important physiological parameters such as respiratory rate without significant added cost or complexity. Such devices are able to provide a better state of patients' health, as the respiratory rate is an important vital sign that is known to be correlated to other physiological conditions such as stress and obesity, and reduce the cost and time to assess the physiological status.

Acknowledgments. Marco A. F. Pimentel was supported by the RCUK Digital Economy Programme [Grant EP/G036861/1]; and Fundação para a Ciência e Tecnologia (FCT), Portugal [Grant SFRH/BD/79799/2011]. Peter H. Charlton acknowledges support from the EPSRC [Grant EP/F058845/1]; and the National Institute for Health Research (NIHR) Biomedical Research Centre based at Guys and St Thomas NHS Foundation Trust and Kings College London. David A. Clifton was supported by a Royal Academy of Engineering Research Fellowship; Balliol College, Oxford; and the Centre of Excellence in Personalised Healthcare funded by the Wellcome Trust and EPSRC [Grant WT 088877/Z/09/Z].

The views expressed are those of the authors and not necessarily those of the EPSRC, NHS, NIHR or Department of Health.

\section{Conflicts of Interest}

Peter H. Charlton has performed consultancy work for Philips Medical Systems (Böblingen, Germany) and OBS Medical Limited (Abingdon, UK).

\section{References}

1. Addison, P.S., Watson, J.N., Mestek, M.L., Mecca, R.S.: Developing an algorithm for pulse oximetry derived respiratory rate $(\mathrm{RR}(\mathrm{oxi}))$ : a healthy volunteer study. Journal of Clinical Monitoring and Computing 26(1), 45-51 (2012)

2. Addison, P.S., Watson, J.N., Mestek, M.L., Ochs, J.P., Uribe, A.A., Bergese, S.D.: Pulse oximetry-derived respiratory rate in general care floor patients. Journal of Clinical Monitoring and Computing 29(1), 113-120 (2015)

3. Bailon, R., Sornmo, L., Laguna, P.: ECG-Derived Respiratory Frequency Estimation. In: Clifford, G.D., Azuaje, F., McSharry, P.E. (eds.) Advanced Methods and Tools for ECG Data Analysis, ch. 8, pp. 215-244. Artech House, London (2006)

4. Bates, A., Ling, M.J., Mann, J., Arvind, D.K.: Respiratory rate and flow waveform estimation from tri-axial accelerometer data. In: 2010 International Conference on Body Sensor Networks, pp. 144-150. IEEE, Singapore (2010) 
5. Cretikos, M.A., Bellomo, R., Hillman, K., Chen, J., Finfer, S., Flabouris, A.: Respiratory rate: the neglected vital sign. The Medical Journal of Australia 188(11), 657-659 (2008)

6. Farrohknia, N., Castrén, M., Ehrenberg, A., Lind, L., Oredsson, S., Jonsson, H., Asplund, K., Göransson, K.E.: Emergency department triage scales and their components: a systematic review of the scientific evidence. Scandinavian Journal of Trauma, Resuscitation and Emergency Medicine 19(1), 42 (2011)

7. Fleming, S.G., Tarassenko, L.: A comparison of signal processing techniques for the extraction of breathing rate from the photoplethysmogram. International Journal of Biological and Medical Sciences 2(4), 232-236 (2007)

8. Garde, A., Karlen, W., Ansermino, J.M., Dumont, G.A.: Estimating respiratory and heart rates from the correntropy spectral density of the photoplethysmogram. PloS One 9(1), e86427 (2014)

9. Goldhaber, S.Z., Visani, L., De Rosa, M.: Acute pulmonary embolism: Clinical outcomes in the International Cooperative Pulmonary Embolism Registry (ICOPER). Lancet 353(2182), 1386-1389 (1999)

10. Jin, A., Yin, B., Morren, G., Duric, H., Aarts, R.M.: Performance evaluation of a tri-axial accelerometry-based respiration monitoring for ambient assisted living. In: Proceedings of the 31st Annual International Conference of the IEEE Engineering in Medicine and Biology Society, pp. 5677-5680 (2009)

11. Karlen, W., Raman, S., Ansermino, J.M., Dumont, G.A.: Multiparameter respiratory rate estimation from the photoplethysmogram. IEEE Transactions on Biomedical Engineering 60(7), 1946-1953 (2013)

12. Karlen, W., Turner, M., Cooke, E., Dumont, G., Ansermino, J.M.: Capnobase: Signal database and tools to collect, share and annotate respiratory signals. In: Annual Meeting of the Society for Technology in Anesthesia (STA), West Palm Beach (2010)

13. Khalil, A., Kelen, G., Rothman, R.E.: A simple screening tool for identification of community-acquired pneumonia in an inner city emergency department. Emergency Medicine Journal 24(5), 336-338 (2007)

14. Knaus, W.A., Wagner, D.P., Draper, E.A., Zimmerman, J.E., Bergner, M., Bastos, P.G., Sirio, C.A., Murphy, D.J., Lotring, T., Damiano, A.: The APACHE III prognostic system. Risk prediction of hospital mortality for critically ill hospitalized adults. Chest 100(6), 1619-1636 (1991)

15. Larsen, P.D., Tzeng, Y.C., Sin, P.Y.W., Galletly, D.C.: Respiratory sinus arrhythmia in conscious humans during spontaneous respiration. Respiratory Physiology \& Neurobiology 174(1-2), 111-118 (2010)

16. Li, B.N., Dong, M.C., Vai, M.I.: On an automatic delineator for arterial blood pressure waveforms. Biomedical Signal Processing and Control 5(1), 76-81 (2010)

17. Li, J., Jin, J., Chen, X., Sun, W., Guo, P.: Comparison of respiratory-induced variations in photoplethysmographic signals. Physiological Measurement 31(3), $415(2010)$

18. Li, Q., Clifford, G.D.: Dynamic time warping and machine learning for signal quality assessment of pulsatile signals. Physiological Measurement 33(9), 1491 (2012)

19. Mackay, D.J.C.: Introduction to gaussian processes. In: NATO ASI Series F Computer and Systems Sciences, vol. 168, pp. 133-166 (1998)

20. Mason, L.: Signal Processing Methods for Non-Invasive Respiration Monitoring. $\mathrm{PhD}$ thesis, University of Oxford (2002) 
